\title{
De Voces y Silencios. Escrituras y Reflexiones en torno a la Indagación de los Aprendizajes de Jóvenes Alumnos en Distintos Contextos ${ }^{1,2}$
}

\section{Andrea Pérez, Silvina Cimolai y Julia Lucas}

\author{
Universidad Nacional de Quilmes, Argentina \\ Universidad Pedagógica de la Provincia de Buenos Aires, Argentina
}

\begin{abstract}
Andrea Pérez es Licenciada en Educación por la Universidad Nacional de Quilmes y Magíster en Gestión Educativa por la Universidad de San Andrés. Es Docente-Investigadora en la Universidad Nacional de Quilmes y en la Universidad Pedagógica de la Provincia de Buenos Aires. Se encuentra finalizando sus estudios de Doctorado en Ciencias Sociales en la Facultad Latinoamericana de Ciencias Sociales gracias a una beca otorgada por CONICET (2009-2011).

Silvina Cimolai es Licenciada en Psicología por la Universidad de Buenos Aires y Magíster en Educación por la Universidad de San Andrés. Desarrolla actividades de Investigación en la Universidad Nacional de Quilmes y es Docente-Investigadora en la Universidad Pedagógica de la Provincia de Buenos Aires. Actualmente se encuentra finalizando sus estudios de Doctorado en Educación en el Instituto de Educación de la Universidad de Londres.

Julia Lucas es docente en escuelas primarias y Licenciada en Educación por la Universidad Nacional de Quilmes donde desarrolla actividades de Investigación. También es Docente-Investigadora en la Universidad Pedagógica de la Provincia de Buenos Aires. Se encuentra finalizando sus estudios de Maestría en Antropología Social en el Instituto de Altos Estudios Sociales.
\end{abstract}

1 Algunos de los temas abordados en este artículo han formado parte de ponencias presentadas en los siguientes eventos: XVI Congreso Mundial de Ciencias de la Educación. Asociación Mundial de Ciencias de la Educación, Monterrey, Nuevo León, México, 31 de mayo a 4 de junio de 2010. VI Jornadas sobre Etnografía y Métodos Cualitativos. Centro de Antropología Social. Instituto de Desarrollo Económico y Social (IDES), 11, 12 y 13 de agosto de 2010. II Congreso Internacional de Investigación y Práctica Profesional en Psicología, XVII Jornadas de Investigación y Sexto Encuentro de Investigadores en Psicología del MERCOSUR. Clínica e Investigación. Contribuciones a las Problemáticas Sociales. Facultad de Psicología-Universidad de Buenos Aires, 22, 23 y 24 de noviembre de 2010.

2 Proyectos de investigación que posibilitaron el presente trabajo: "Sujetos, Aprendizajes y Contextos", dirigido por Ricardo Baquero en la Universidad Pedagógica de la Provincia de Buenos Aires, y "Escuela, Diferencia e Inclusión”, dirigido por Silvia Porro en la Universidad Nacional de Quilmes (Argentina). 


\title{
De Voces y Silencios. Escrituras y Reflexiones en torno a la Indagación de los Aprendizajes de Jóvenes Alumnos en Distintos Contextos
}

\begin{abstract}
Resumen
El artículo reflexiona a partir de dos líneas de trabajo desarrolladas por un programa de investigación en curso en la Argentina: por un lado, se interesa por conocer las particularidades que adoptan los aprendizajes que los estudiantes construyen en contextos escolares y extraescolares; por otro lado, se interesa por destacar las voces de los estudiantes de acuerdo con una tradición teóricometodológica que intenta posicionarlos activamente en lo que respecta a las investigaciones educativas. A partir de un abordaje cualitativo y, en particular, de algunos recursos propios de los enfoques etnográficos, se propuso a los estudiantes de una escuela secundaria la realización conjunta de un "diario de aprendizajes". Las sensaciones, emociones y reflexiones, pero, en particular, las voces y los silencios de los estudiantes, pasaron a un primer plano, para ponerse en juego en tanto aspectos clave de los procesos de aprendizaje escolares y extraescolares, y de la misma investigación educativa.
\end{abstract}

Palabras clave: escuela secundaria, contexto de aprendizaje, investigación pedagógica, relación alumno-escuela, educación extraescolar 


\title{
About Voices and Silences. Writings and Reflections on the Inquiry into the Learning of Young Students in Different Contexts
}

\begin{abstract}
The work reflects upon two research lines being carried out in Argentina in a research programme: On the one hand, it focuses on studying the particular characteristics in which students build up their learning within school and out-of-school contexts; on the other hand, although closely related to this, it focuses on highlighting the students' voices according to a theoretical and methodological tradition which attempts to position students actively in educational research. Within a cualitative etnographical approach, the research team developed a "learning diary" with students' groups of a Secondary School. Students' feelings, emotions, thoughts, voices, and silences become outstanding and key factors around school and out-of-school learning processes.
\end{abstract}

Keywords: Secondary School, learning context, educational research, pupil-school relationship, out-of-school education 


\section{Presentación}

El presente artículo tiene su origen en un programa de investigación desa- rrollado en escuelas secundarias de Quilmes, distrito ubicado en los alrededores de la Ciudad de Buenos Aires (Argentina), es decir, la región urbana también conocida como "conurbano bonaerense".

Entre los temas que aborda el programa de investigación se encuentran, por un lado, el interés por conocer las particularidades que adoptan los aprendizajes que los estudiantes construyen en contextos escolares y extraescolares, intentando una aproximación a la complejidad que cada una de estas instancias implica en sus experiencias cotidianas. Por otro lado, el foco está puesto en indagar las formas en que se pueden promover posiciones alternativas de los estudiantes en los procesos de construcción de conocimientos.

Con respecto al primer punto, existe una tradición de estudios interesados en las continuidades y discontinuidades entre los distintos contextos de aprendizaje, que marcan un camino fecundo para la indagación y comprensión de los sentidos, formas de participación y apropiación de los aprendizajes que los sujetos construyen de acuerdo con el contexto en el que se encuentran. En cuanto al segundo punto, consideramos los aportes de los estudios que abordan "las voces del alumnado", de acuerdo con una tradición teóricometodológica que busca posicionarlos activamente en lo que respecta a las investigaciones escolares en las que se les solicita participar. Se trata de un abordaje centrado, entre otros aspectos, en la generación y valoración de posicionamientos críticos por parte de los actores involucrados, y una deliberada consideración de sus opiniones en las potenciales orientaciones que puede tomar la indagación, como así también las prácticas escolares en su conjunto.

Anima nuestro trabajo una fuerte convicción en torno de la necesidad de que los dispositivos institucionales tradicionales del sistema educativo sean puestos en discusión, no solo en los ámbitos académicos, sino también -y fundamentalmente- en los ámbitos en los que los procesos de aprendizaje de los sujetos en edad escolar son puestos en juego (contextos escolares y extraescolares). Los adultos se ven, asimismo, interpelados y, en ocasiones, ponen en cuestión o modifican ciertos supuestos tradicionales respecto del alumnado. Se trata de la apertura a reformular ciertas estructuras y prácticas tradicionales (Rudduck y Flutter, 2007). Se trata, en definitiva, de un modo de adecuar los procesos educativos a los nuevos y cambiantes tiempos, no solo desde el punto de vista de las reformas políticas educativas sino, fundamentalmente, a partir de la potencialidad comprometida en las pequeñas pero 
intensas transformaciones cotidianas, de carácter gradual y singular, que solo los actores más directamente involucrados pueden generar a un más largo plazo.

Consideramos que la carga emotiva involucrada en los procesos de construcción de conocimientos y, más generalmente, en las trayectorias formativas de los sujetos, constituye un plano central a tener en cuenta si de transformaciones se trata. A pesar de la importancia de los lineamientos curriculares y de las políticas educativas en general, a pesar, incluso, de los esfuerzos generados por los referentes de cada institución para mejorar las prácticas escolares, los protagonistas ineludibles en lo que a la emergencia de conocimientos respecta son los alumnos y, más específicamente, sus procesos internos, en permanente interacción con los objetos culturales y los otros sujetos. Sin embargo, estos procesos internos atravesados por los alumnos no siempre son considerados a la hora de tomar decisiones respecto de ellos.

En este marco, el trabajo se propone compartir una experiencia de trabajo de campo a la que llamamos "diarios de aprendizaje" — realizada en una de las instituciones estudiadas-, como también exponer los resultados preliminares de la misma. El trabajo con los diarios de aprendizaje fue ideado y desarrollado con el fin de conocer apreciaciones de los estudiantes en torno de los aprendizajes escolares y extraescolares, a la vez que favorecer instancias de participación de los mismos, en línea con los temas de interés del programa de investigación.

Para ello, el artículo se organiza del siguiente modo: en los dos primeros apartados, "Aprendizajes en contextos escolares y extraescolares" y "Las voces de los alumnos y su 'potencial transformador'”, presentamos un breve estado del arte con el fin de introducir al lector en los aportes teóricos que orientan nuestro trabajo.

Seguidamente, exponemos las características del abordaje metodológico utilizado durante nuestra indagación empírica. Dicho abordaje, de carácter cualitativo, fue enriquecido por los aportes de los enfoques etnográficos mediante recursos tales como la observación participante, el registro sistemático de lo observado, lo leído y lo escuchado, la focalización en las "voces nativas", etc.

Luego de los aspectos metodológicos dedicamos dos apartados a reflexionar acerca de algunos resultados preliminares a la luz de los aportes teóricos anteriormente desarrollados; finalizamos el artículo con algunas reflexiones e interrogantes que pretenden invitar a pensar nuevas perspectivas de intervención y análisis en el marco de nuestra labor como docentes e investigadores. 


\section{Aprendizajes en Contextos Escolares y Extra-escolares}

En los espacios destinados a la educación formal, los estudiantes participan de situaciones escolares en compañía de pares y referentes adultos de la escuela, poniendo en juego actividades y contenidos específicamente pedagogizados y legitimados a través de las prescripciones curriculares previstas para cada año. Por otro lado, en contextos extraescolares como los que brindan las familias, los barrios, las organizaciones sociales, entre otros, los sujetos despliegan, crean y recrean instancias más o menos formales, más o menos explícitas, que, en todos los casos, y aunque con rasgos claramente diferentes a los de la institución escolar, constituyen instancias fundamentales en la construcción de sus trayectorias de vida y sus aprendizajes. Es claro que, a los fines de la conformación de subjetividades y de los sentidos otorgados a las distintas prácticas sociales, todos los contextos - y todo lo que ellos involucran - constituyen una misma y compleja trama; también es claro que ciertas indagaciones y distinciones permiten hallar continuidades y discontinuidades de suma importancia a los fines de analizar y contribuir a la comprensión de los procesos de aprendizaje desde una perspectiva situada. En el presente apartado intentaremos exponer algunas de las tensiones que aportan a esta discusión.

Existe una variada tradición académica en torno a los estudios que se preguntan por la conveniencia o inconveniencia de incluir los conocimientos y formas de aprender que niños y adolescentes construyen en sus contextos de crianza, teniendo en cuenta la especificidad de aquello que se ofrece desde las experiencias escolares. El debate se erige en torno a qué incluir - si conocimientos o formas de aprender - así como de la validez misma de hacerlo. En algunos casos el problema se plantea de manera polarizada, dando cuenta de una reivindicación algo ingenua de las prácticas no escolares sin su correspondiente crítica o análisis - como, por ejemplo, la situación real de las condiciones de crianza o de trabajo usuales-. En otros casos, el foco está puesto en una valoración per se de las formas de construcción de conocimientos descontextualizadas o promotoras de un pensamiento abstracto que portarían, como efecto positivo, las prácticas escolares más "felices".

En un análisis detallado de los estudios sobre aprendizajes escolares y extraescolares (Cimolai, Lucas y Pérez, 2011) advertimos la existencia de, al menos, las siguientes líneas de investigación, a las que hemos agrupado de acuerdo con los temas centrales sobre los que trabajan. En un primer grupo, se encuentran los trabajos de Bernstein (1971), Labov (1969), Cazden (1991), 
Wells (1986) y, en el ámbito local, Borzone y Rosemberg (2000), Gandulfo (2007), entre otros, quienes abordan los contextos escolares y extraescolares de aprendizaje analizando sus diferencias en términos de los usos del lenguaje y las distintas formas de participar e interactuar que tienen los sujetos; en segundo lugar, pueden señalarse estudios como los de Limón y Carretero (1997) y Candela (2006), entre otros, más orientados a estudiar cómo son utilizados - o cómo pueden ser utilizados - los conocimientos y formas de aprender extraescolares en la institución escolar; un tercer grupo sería el representado por Carraher, Carraher y Schlieman (1998), Ferreiro (1986), Ferreiro y Teberosky (1979) o Andrews y Yee (2006), quienes han puesto el foco en cómo se construyen, en contextos extraescolares, los conocimientos tradicionalmente identificados como "contenidos escolares"; finalmente, se hallan los trabajos que tienen en común poner el foco de análisis en los espacios de interacción entre las escuelas y las familias. Aquí se encuentran, por ejemplo, los desarroIlos de Moll y Greenberg (1990), Hughes (2006a, 2006b), Jasis y Ordoñez-Jasis (2004) y, en el ámbito local, Elichiry, Scavino y Arrúe (2005), y Elichiry et al., (2008), entre otros.

El análisis de estas investigaciones muestra que los diversos aprendizajes y contextos estudiados suelen ser caracterizados en términos de ruptura, y las posiciones subjetivas del alumnado son leídas en términos de actividad/ pasividad. En este sentido, cabe advertir la necesidad de observar con mayor grado de detalle los matices que pudieran presentarse en las relaciones entre los diversos contextos, teniendo en cuenta los altos grados de dinamismo que existen entre los mismos.

Otro de los aspectos observados en estas investigaciones pone de manifiesto que las indagaciones suelen estar definidas a partir de los conocimientos, formas de aprender y contenidos que son tradicionalmente escolares, desestimando la indagación de otros contenidos o aspectos de los aprendizajes que podrían ser relevantes.

Finalmente, cabe mencionar la vinculación que, en general, hacen estos trabajos entre la definición del problema de investigación y la focalización en grupos poblacionales provenientes de sectores socioeconómicamente desfavorecidos o minorías étnicas. Existe un supuesto generalizado que indica que este tipo de indagaciones podría proveer conocimientos y estrategias para contribuir a la disminución del fracaso escolar en dichos sectores.

A pesar de que estas discusiones no pueden ser profundizadas aquí, brindan un panorama de la riqueza que aportan los estudios interesados en los aprendizajes construidos en distintos contextos, dando cuenta de dos aspectos 
íntimamente relacionados entre sí: en primer lugar, el creciente valor otorgado por la investigación académica a la construcción de conocimientos fuera de la institución que, durante muchas décadas, se asumió como monopólica en lo que a los procesos de construcción de aprendizajes refiere; en segundo lugar, la creciente aproximación a estos procesos a través de los aportes provenientes de distintas perspectivas teóricas y metodológicas. No solo se destaca la fuerte impronta de las perspectivas asociadas a la psicología del desarrollo o del aprendizaje - tradicionalmente preocupadas por el tratamiento otorgado a los aprendizajes desde el punto de vista escolar-. También se destacan los estudios de índole discursiva, sociológica y etnográfica, los cuales brindan nuevas herramientas que contribuyen a una mirada más integral de la compleja realidad a la que nos enfrentamos los investigadores interesados en problemas educativos actuales.

En la actualidad, se advierte que los sentidos y prácticas que el estudiantado experimenta en sus vidas cotidianas distan mucho de coincidir con los sentidos y las formas en que los sistemas educativos han montado históricamente sus dispositivos institucionales, esto es, su autoridad en relación con los saberes y valores sociales, su formato áulico y curricular, la orientación brindada a los vínculos interpersonales, el "disciplinamiento de los cuerpos" y el predominio de conocimientos atribuidos a la ciencia, etc. En relación con esto, existe una serie de estudios que señala que los estudiantes suelen transitar contextos extraescolares con márgenes mucho mayores de autonomía que los que mantienen en los contextos escolares, en los que, contrariamente, predomina una visión infantilizante y heterónoma del estudiantado -independientemente de su edad- (MacBeath, Demetriou, Rudduk y Myers, 2003) ${ }^{3}$. En este sentido, tal como señala Kaplún (2004) la escuela parece destinar grandes esfuerzos formales por incluir al estudiantado en el sistema, mientras que los excluye culturalmente en su propuesta, profundizando de ese modo la separación entre el mundo escolar y el extraescolar.

Las representaciones acerca de la juventud centradas en el "déficit" es un tema abordado por Chaves (2005). Esta autora destaca la existencia de diferentes formaciones discursivas - naturalista, psicologista, sociologista, culturalista, de la patología social, etc.- - que buscan definir a este amplio grupo etáreo a

3 Cabe recordar que esta concepción infantilizante y heterónoma de quienes se encuentran en el "lugar del alumno" ha contribuido a justificar la obligatoriedad escolar moderna (Gélis, 1994; Aries, 1986), como así también la deliberada legitimación y especialización de los conocimientos allí impartidos. 
partir de una concepción esencialista, del "ser en sí mismo". Estas representaciones comparten una lógica que caracteriza a los sujetos desde la falta, la ausencia y la negación, como si estos aspectos constituyeran "su esencia". En esa línea, suelen girar en torno al joven en tanto "ser inseguro de sí mismo", "ser en transición", "ser no productivo", "incompleto", "desinteresado o sin deseo", "desviado", "peligroso", "victimizado", "rebelde o revolucionario", "ser del futuro". En el marco de un conjunto mayor de cuestionamientos a la lógica de la escuela tradicional, el acento que esta ha puesto en "lo que al alumno le falta para" (ser adulto, ser experto, o simplemente "ser") constituye uno de los aspectos más criticados por los estudios centrados en las voces de los alumnos (Chaves, 2005, pp. 197-201).

\section{Las Voces de los Alumnos y su "Potencial Transformador"}

Las características que adoptan las voces y las posiciones del alumnado en las experiencias escolares y en la investigación educativa se han convertido en los últimos tiempos en un núcleo común de intereses a indagar, acompañado de una renovada necesidad por definir los términos de esa indagación. Dentro de este núcleo de intereses se reconoce una serie de iniciativas de investigación preocupadas por explorar las formas alternativas de participación de las voces del estudiantado, como también su mayor protagonismo. Según expresan autores como Rudduck y McIntyre (2007), Rudduck (2006) y Fielding (2004), la mayor parte de la tradición en investigación que ha contemplado las voces de los alumnos no ha podido ir más allá de considerar a los niños y adolescentes como objetos o fuentes de información en las investigaciones, aunque cabe mencionar que muchas de ellas —-incluyendo desde estudios sobre "representaciones" hasta análisis estadísticos de opiniones - han contribuido a aportar aspectos clave en torno de estas voces. Milstein (2006) también realiza una advertencia en este sentido: para esta autora, si bien existen distintas corrientes en la investigación educativa - o la investigación en general- que han tomado como objeto/sujeto de estudio a las escuelas o al alumnado, no han sido sus voces las que comúnmente se han indagado. En palabras de esta autora,

[...] se narran situaciones en las que están involucrados, se describen formas de buen y mal trato, se apela a su protección y resguardo, se vela por su cuidado, etc.; pero sus historias y vivencias narradas, sus percepciones e interpretaciones no se incluyen como parte de lo que se denomina 
"perspectiva de los actores". Se habla de los chicos y de las chicas pero, lo que ellos y ellas cuentan, no figura en el mismo nivel que lo que cuentan los adultos como constitutivo de las interacciones sociales. Esto indica que no se advierte que las historias que ellos narran, también modelan las experiencias vividas que se están etnografiando. (Milstein 2006, p. 50)

El creciente desarrollo de estudios que se ocupan de considerar a los estudiantes como sujetos activos involucrados en la investigación se inicia a partir de la tradición de investigación-acción e investigación docente basada en los principios de inclusión y participación (Fielding y Bragg, 2003). Este posicionamiento va de la mano de una corriente teórica proveniente del campo del currículum, interesada por indagar y resolver el hiato existente entre la teoría y la práctica educativa, a partir de una perspectiva atenta a los procesos más que a la lógica "medios-fines" tradicional en dicho campo (Stenhouse, 1991). Se busca fomentar que los mismos alumnos trabajen activamente y tomen decisiones junto con sus docentes en actividades de indagación que tengan como objetivo mejorar los procesos de aprendizaje. Se trata de estudios habitualmente llamados "investigaciones participativas", en los que los estudiantes se comprometen en un proceso como informantes y como "investigadores", y los docentes (apoyados por los investigadores) actúan como facilitadores durante el desarrollo de la experiencia.

Harris y McGregor (2007) sintetizan el interés de las investigaciones centradas en "las voces" del alumnado como la necesidad de considerar a los estudiantes de un modo distinto del tradicional, en tanto son estas voces las que permiten

[...] reevaluar sus capacidades y revisar y cambiar distintos aspectos de la organización, las relaciones y las prácticas escolares de manera que reflejen lo que los jóvenes son capaces de ser y de hacer. [...] se indica que tenemos que tomar en serio lo que nos dicen los alumnos sobre sus experiencias como aprendices en los centros, lo que les dificulta el aprendizaje y lo que les ayuda a ello. [...]. Como "testigos expertos", dan su visión de las condiciones de aprendizaje que permiten contemplar alternativas como primer paso de un cambio fundamental. (Harris y McGregor, 2007, p. 15)

Este interés por revisar las formas de inclusión de las voces de los alumnos en las investigaciones educativas tiene sus principios en un movimiento educativo más general de búsqueda de formas alternativas de inclusión de dichas 
voces en la vida cotidiana escolar. Rudduck y Mclntyre (2007) identifican una serie de principios y presuposiciones - sintetizados a continuación - que han influido en la búsqueda de una redefinición de estos acercamientos.

- El principio de desarrollo democrático, donde los procesos de escolarización asumen su responsabilidad en contribuir a preparar a niños y adolescentes para una vida en sociedad. Algunas investigaciones educativas (McGregor, 2005) han establecido estrechos lazos entre estudios sobre formación ciudadana en la escuela, por un lado, y los procesos de consulta y participación de los alumnos dentro del sistema educativo, por el otro.

- $\quad$ El principio de los derechos de niños y adolescentes, incluyendo aquellos derechos que tienen por destinatarios a los miembros de la comunidad escolar. Este principio conlleva la revisión de las formas de participación que se promueven en la escuela.

- La preocupación por los cursos a dar al desarrollo personal y social de los niños, en los que los procesos de consulta y participación construirían interacciones basadas en el respeto y la confianza.

- La idea de que el "escuchar" las voces de los alumnos puede promover un mayor compromiso de los mismos con respecto a sus aprendizajes y los de sus pares: ciertas formas de inclusión de las voces han demostrado generar un sentido de agencia de parte de ellos, impactando positivamente en sus rendimientos escolares.

Existen diferentes perspectivas que contemplan la inclusión de la voz de los alumnos en los procesos de investigación y en la toma de decisiones en contextos escolares. En el marco de la corriente denominada student voice, Fielding (2004) ha desarrollado una tipología que identifica cuatro grandes posicionamientos de investigación: se los considera como fuentes de información, como participantes activos de las discusiones, como coinvestigadores y como investigadores. Sostiene que esta tipología no es jerárquica, sino que depende de los objetivos que guían la actividad en la que las mismas están siendo consideradas.

Los trabajos centrados en las voces de los alumnos consideran a los docentes como componentes fundamentales respecto del "potencial transformador" de la voz de los alumnos (Rudduck y Flutter, 2007). A diferencia de la lógica del déficit, el énfasis está puesto aquí en una posibilidad de transformación de las prácticas y perspectivas tradicionales tan arraigadas en los sistemas educativos. Desde esta perspectiva, el trabajo cotidiano se torna más complejo e 
incierto, en tanto que involucra el ensayo gradual de distintas alternativas de trabajo conjunto con los alumnos y los demás docentes de la institución. Los docentes deben fomentar la participación activa de los alumnos en la toma de decisiones respecto de distintos asuntos de la vida escolar, en particular, en actividades de indagación que tengan como objetivo mejorar los procesos de construcción de aprendizajes. De este modo, estudiantes y docentes asumen un compromiso en el marco de un proceso educativo que los considera como "informantes" a la vez que como "investigadores". Los estudiantes son considerados testigos privilegiados de los procesos de aprendizaje escolar por lo que, para los docentes, dar lugar a que el alumnado se exprese y posicione al respecto puede resultar, entre otras cosas, en una percepción más abierta de las capacidades de los alumnos, una creciente capacidad de ver lo conocido desde distintas perspectivas, una mejor disposición para modificar formas de pensar y prácticas, un renovado interés por la enseñanza y un plan práctico para mejorarla (Rudduck y Flutter, 2007).

Hasta aquí hemos presentado un breve estado del arte que enmarca teóricamente nuestra indagación empírica y nuestras perspectivas de trabajo. A continuación, nuestro desarrollo se centrará en los aspectos metodológicos de dicha indagación, para pasar luego a las reflexiones suscitadas a partir de la misma.

\section{Aspectos Metodológicos de Nuestro Trabajo}

Si bien el programa de investigación realizó indagaciones en distintas escuelas, la experiencia compartida en el presente trabajo refiere solo a una de ellas: una escuela secundaria de la localidad de San Francisco Solano del distrito de Quilmes. La elección es esta escuela fue realizada a partir de la necesidad de trabajar con jóvenes de escuelas secundarias que atendieran a sectores socioeconómicamente vulnerables, donde docentes y directivos mostraran cierta preocupación por implementar proyectos que promovieran mayores grados de participación del alumnado. A partir del vínculo establecido con informantes clave - en este caso, directivos con los cuales el equipo de investigación ya venía trabajando- nos acercamos a esta escuela.

El primer ingreso a esta institución se hizo a través de un contacto con el director de la misma, a quien entrevistamos en dos oportunidades con el fin de contarle nuestros objetivos, conocer sus apreciaciones y caracterizaciones de la institución y, finalmente, obtener su aval para realizar nuestro trabajo de campo en la misma. 
Posteriormente, concertamos entrevistas con otros adultos que se desempeñaban en la institución: cuatro docentes y tres preceptores. El propósito de las mismas era lograr un primer relevamiento de las actividades que los adolescentes realizaban cotidianamente. Estas indagaciones permitieron elaborar una aproximación a cómo eran consideradas, desde el punto de vista de los adultos consultados, las actividades extraescolares de estos adolescentes, como así también registrar las formas en que los docentes decían poner en diálogo los aprendizajes escolares y extraescolares de sus estudiantes. Los relatos de estos actores mostraron una diversidad de posiciones. Luego de la sistematización de las entrevistas, y a fin de utilizar esta información para la indagación de los alumnos, distinguimos las siguientes categorías. Los aprendizajes extraescolares eran concebidos por los docentes entrevistados como:

- elementos disruptivos de la dinámica del aula;

- elementos empobrecedores de ciertos aprendizajes escolares;

- temáticas consideradas pertinentes para incluir en la planificación curricular;

- aliados para potenciar aprendizajes e intereses escolares;

- $\quad$ modos de acreditación de conocimientos o aprendizajes escolares.

En una tercera instancia, y frente a la necesidad de conocer en profundidad las actividades, conocimientos, valoraciones y formas de aprender de los adolescentes, decidimos concretar una serie de actividades teniendo en cuenta las indagaciones previas. Propusimos a los referentes institucionales de la escuela el trabajo con los estudiantes. Planteamos entonces la posibilidad de que cada estudiante realizara la escritura de lo que denominaríamos un "diario de aprendizajes", el cual tenía dos propósitos: por un lado, explorar nuevas estrategias de trabajo investigativo con adolescentes; por otro lado, y en línea con lo anterior y con nuestro marco teórico, favorecer la creciente participación de los estudiantes. Gracias a la activa colaboración de los docentes, dos de ellos habilitaron dos horas de sus respectivas clases semanales para estos encuentros.

En un lugar donde los silencios y la calma predominaban por sobre el desorden y el bullicio, el diario de aprendizajes parecía ser un instrumento de indagación y participación potente para favorecer la irrupción de la palabra de quienes se autocaracterizaban - y también eran caracterizados por los adultos de la escuela - como "callados" y "tímidos". El diario fue tomando 142 I forma de manera muy gradual y atenta a lo que iba ocurriendo en el trans- 
curso de los encuentros que teníamos con ellos una vez por semana (en total, se desarrollaron doce encuentros). En términos generales, la asistencia de los jóvenes a la escuela era regular, a excepción de casos puntuales de jóvenes que presentaban un alto grado de inasistencia a clases.

Concretamente, el desarrollo de la experiencia consistió en una serie de encuentros - registrados con un grabador de audio- entre integrantes del equipo de investigación y los 35 alumnos del segundo año del turno tarde de la escuela (todos ellos de alrededor de 17 años de edad). En los encuentros se proponía una determinada consigna a partir de la cual se solicitaba redactar, en sus respectivos cuadernos, anotaciones y reflexiones personales. En los siguientes encuentros, dichas anotaciones y reflexiones eran puestas en común en el marco del grupo (cabe aclarar que los referentes de la institución no formaron parte de estas reuniones).

Dado el carácter cualitativo de nuestro estudio, el trabajo fue desarrollado a partir de nuestros supuestos, lecturas iniciales, y datos brindados por los adultos y adolescentes de la institución. Los "ajustes" fueron una constante. Brindamos especial atención a lo que ocurría en cada uno de los encuentros, puesto que esas eran las instancias decisivas que marcarían cada uno de los pasos siguientes. De este modo, el aula se convirtió en un espacio de encuentro y diálogo en el que los alumnos compartían lo que habían escrito durante la semana a partir de los temas planteados, pero también de nuevas consignas ideadas por ellos de acuerdo con cómo se iba desarrollando el proceso. En general, estas consignas apelaron a movilizar y poner de manifiesto asuntos personales, muchas veces - aunque no siempre - ligados a los aprendizajes, pero desde perspectivas claramente distintas de las relacionadas con la institución escolar. Esta orientación del trabajo hacia la indagación de sensaciones y reflexiones personales tuvo la intención de lograr que los alumnos se posicionaran de modos diferentes de los habituales en la escuela, buscando un creciente compromiso con la actividad del grupo.

A modo de ilustración, podemos mencionar que, entre las consignas que sirvieron de "disparadores" para abordar los distintos encuentros, se hallan las siguientes:

- ¿Quién soy?

- Describo una clase que me guste mucho o que no me guste y justifico por qué

- Describo un día de mi vida

- En qué siento que soy muy bueno o sobre qué siento que sé mucho

- Elijo una actividad que me guste hacer y cuento cómo la aprendí 
- ¿ ¿Qué me gusta de la escuela y qué no?

- ¿ ¿Qué materias me gustan más y por qué?

Cabe señalar que el abordaje cualitativo de nuestra propuesta se vio enriquecido por aportes propios de la etnografía. La realización de entrevistas o encuentros se vio favorecida por las estancias prolongadas del equipo de investigación en diversos eventos y acontecimientos de la vida cotidiana escolar, por la observación participante y, principalmente, por la necesidad de reflexión constante sobre los supuestos de quienes llevamos adelante esta investigación.

El análisis de los datos obtenidos a partir de la escritura de estos diarios y de los encuentros semanales se realizó sobre la base de las desgrabaciones de ambos elementos. Desde el equipo de investigación se propusieron algunos ejes analíticos para la lectura de los mismos, aunque otros fueron surgiendo en el transcurso de la investigación. Así, por ejemplo, en un primer momento, se utilizaban aprendizaje escolar y extraescolar como categorías homogéneas en sí mismas, cuando finalmente lo que se encontró en la indagación -como se verá luego - fue una suma de continuidades y discontinuidades en ambos tipos de aprendizaje, así como diferenciaciones dentro de los mismos ámbitos.

Para finalizar este apartado, y antes de continuar abordando lo producido específicamente a partir de los diarios de aprendizaje, cabe señalar algunos rasgos del contexto en el que trabajamos, como así también otras notas de campo llevadas adelante por el equipo de investigación.

En primer lugar, podemos decir que desde un principio nos llamó la atención el habitual silencio - ya mencionado- que caracterizaba a esta escuela. Desde nuestra mirada, influida por experiencias previas en otras instituciones, resultaba extraño que una escuela de adolescentes no estuviera plagada de ruidos, conversaciones, discusiones, retos o paredes pintadas.

Por otro lado nos resultaba llamativo el hecho de que en varias oportunidades en que nos presentamos en la escuela para continuar nuestras tareas de campo, varias aulas se encontraban vacías. En dichas oportunidades, quienes nos atendían en la institución nos informaban que, dada la frecuente inasistencia de algunos profesores, los alumnos entraban más tarde o se iban más temprano de la misma, algo que, en ocasiones, veíamos como una amenaza a la continuidad de nuestro trabajo.

Pero lo que más llamó nuestra atención fue el modo en que casi todos los alumnos hacían referencia a sí mismos cuando les preguntábamos cómo se percibían como tales. Ejemplo de esto son las siguientes expresiones: "Soy muy callada, solamente hablo con algunos compañeros con los que tengo 
confianza"; "Soy el tipo de personas que son callados y tímidos pero sólo con las personas que no me conocen, pero si me dan tres palabras y una sonrisa, listo, ya se me va la timidez y me comporto natural".

Como puede advertirse, el silencio y las ausencias se constituyeron en variables fundamentales a tener en cuenta durante las tareas de campo. ¿Qué concepciones acerca de los sujetos entran en juego en estos escenarios? ¿Qué intervenciones realizar como referentes adultos? ¿Qué posicionamientos asumir en el marco de nuestras prácticas pedagógicas?

Como ya fue anticipado en la presentación, a continuación nos detendremos en nuestra experiencia de indagación, haciendo especial hincapié en estas cuestiones.

\section{Voces en Acción: Nuestra Experiencia con los Diarios de Aprendizaje}

El ejercicio de escribir en torno a temas comunes y compartir luego lo escrito con el resto del grupo fue inicialmente conceptualizado por algunos estudiantes como un condicionante para su efectiva participación. Durante los primeros diálogos surgieron afirmaciones tales como "Yo no quiero participar, aunque no figure mi nombre todos se van a dar cuenta que fui yo por mi letra... es re fea".

Estas situaciones nos condujeron a repensar y reformular algunas estrategias, ya que en varias oportunidades hubo quienes no escribían. Nos encontramos en una disyuntiva acerca de qué hacer, es decir, si seguir insistiendo en la escritura a riesgo de perder participaciones genuinas, o si priorizar la participación, en desmedro de las instancias de escritura.

La resolución tomada por el equipo de investigación se orientó a esta última alternativa. Sabíamos que trabajar en un contexto como el escolar podía facilitar algunos procesos pero también generar serios obstáculos, tales como la inhibición frente a la pregunta de un adulto/docente, la percepción de sentirse evaluado frente a la solicitud de escritura, o la dificultad de compartir aspectos personales en una institución como la escuela, en la que los conocimientos escolares son sobrestimados en relación con las sensaciones y valoraciones propias. Fue así como los encuentros fueron tomando forma de conversaciones más que de entrevistas grupales, dando lugar a una gradual y creciente confianza de todos los que participaban del grupo de trabajo, lo que fue generando una también creciente producción de intervenciones, tanto escritas como orales. Este espacio se fue convirtiendo en una instancia para 
que los adolescentes hablaran y pensaran críticamente respecto de distintas cuestiones; para nosotros, investigadores, devino en la necesidad de poner en diálogo las posiciones, concepciones y representaciones del equipo de investigación con el contexto escolar en el que se llevaba adelante el trabajo de campo y las voces del alumnado.

Asimismo, la posibilidad de que el alumnado pudiera registrar, pensar y repensar sus propios aprendizajes, devino en una nueva forma de poner en diálogo las diversas voces de la experiencia escolar. Uno de los aspectos a destacar es que, en momentos en que existe un imaginario social tendiente a considerar a los adolescentes como apáticos, desinteresados, etc. surgieron planteamientos - de parte de los alumnos participantes - fuertemente críticos respecto de los adultos: señalaban que las culturas y situaciones barriales, locales o familiares condicionaban la cantidad y calidad de los aprendizajes, en tanto eran aspectos que influían en las decisiones de sus docentes de "bajar el nivel de los contenidos" escolares impartidos, con todas las consecuencias que ello puede implicar en términos sociales y subjetivos. En este sentido cabe destacar que esta percepción de los estudiantes se condecía con varios de los relatos de sus docentes, quienes efectivamente señalaban que los aprendizajes extraescolares de sus alumnos operaban como factores que condicionaban el aprendizaje de los contenidos escolares. A continuación, reproducimos un fragmento de una de las conversaciones ${ }^{4}$ :

Alumno 2: En otras escuelas te dan más y acá no

Alumno 3: Hay materias que no aprendemos nada como TIC. Por suerte no la tenemos en tercero, sino no, no sabemos nada

Entrevistadora: $Y$ eso que vos decías de que no nos dan nada... Ustedes qué piensan de eso... ¿qué significa "que no te dan nada"?

Alumno 3: Que no te enseñan nada...

Entrevistadora: $Y$ ¿por qué pensás que pasa eso?

Alumno 2: Porque tienen vagancia

Alumno 3: Y por la forma de la escuela también

Entrevistadora: ¿Qué forma tiene la escuela?

Alumno 3: En esta escuela a la noche vienen chicos que son más... No les interesa mucho... Los profesores se dan cuenta... y ya está...

$4 \quad$ Hemos optado por mantener, en las trascripciones de las conversaciones, las expresiones orales locales utilizados por los participantes: en las transcripciones se advertirán acentos erróneos desde el punto de vista ortográfico, pero necesarios para reflejar la pronunciación utilizada habitualmente en algunas regiones de la Argentina. 
Alumno 2: Encima te regalan la nota....

Entrevistadora: ¿Cómo es eso?

Alumno 2: Y... hacés dos trabajitos más y te aprueban

Entrevistador: ¿Está bueno eso? ¿Qué piensan de eso ustedes?

Alumno 3: Está bueno, pero así no aprendés nada

En otro momento, cuando se les pidió que eligieran una clase y la caracterizaran, los estudiantes apelaban a descripciones del tipo "todos los alumnos están callados"; "el lugar es la escuela, el salón donde estamos todos muy callados"; "lo que tiene es que somos pocos y cada uno está en la suya, no tenemos diálogo ni nada". Según las voces de estos jóvenes, "estar callado" podía hacer referencia a dos tipos de situaciones de clase: aquellas clases donde se sentían interpelados o implicados desde la actividad propuesta o, por el contrario, aquellas donde el único que hablaba y decidía qué y cómo sería la clase era el docente.

Respecto de este último tipo de situaciones, los jóvenes hicieron referencia constante a una clase en particular - la clase de inglés-, que tomamos aquí para ilustrar: "nadie entiende a la profesora. Ella dice: hagan la tarea, y se pone a hablar... Después dice: entreguen la tarea, y se la pasó hablando de la tarea y qué sé yo"; "en inglés no entendemos nada. Nos habla todo el tiempo en inglés"; "entra, no saluda ni nada, pone tarea. Ninguno del salón le presta atención y ella como siempre tira palazos: que somos el peor salón y que a ella no le importa si aprobamos o no".

Estas descripciones se complementan con otros momentos del relato, donde los alumnos y alumnas narran eventos, situaciones o simplemente "artes de hacer" - en términos de Certeau (1979) - que permiten visualizar aquello que los alumnos efectivamente hacen a pesar de la aparente inactividad o apatía: "todos los alumnos están callados. Los únicos que hablamos somos nosotros, pero lo hacemos en voz baja. Todos los demás están haciendo la tarea. Nadie entiende a la profesora"; "estoy en la clase de inglés, hablando con mis amigos, porque no me llama la atención aprender inglés. La profe nos reta y nos dice que hagamos la tarea, que nos callemos. Y nos callamos pero seguimos hablando y hacemos como que nos callamos. Los demás también están hablando en voz baja, para que no los escuche la profe"; "durante la hora de inglés con dos amigos y amigas jugamos y hablamos y así pasamos rápido la hora. Bueno, en fin, la hora de inglés no me gusta para nada".

Estos actos de habla ocurridos cuando lo que se pide es silencio refieren a lo que el alumnado efectivamente hace durante las horas de clase y remite 
a la vieja tradición en educación acerca del "oficio de alumno". Este "arte de hacer" permite vislumbrar aquello que se hace con los discursos hegemónicos; desde el punto de vista escolar, permite reflexionar en torno al diálogo que se establece - o no- entre las voces del alumnado y las voces docentes -aquellas que, por tradición, están autorizadas para decidir qué, cuándo y cómo hablar-.

Tanto en la realización de actividades conjuntas (entre el equipo de investigación, los estudiantes, los docentes, los preceptores y el director) así como en las entrevistas realizadas, los relatos de los alumnos y las alumnas remitían constantemente a la posibilidad de hablar o mantenerse callados en el salón de clases. La forma en que aparecían sus voces y las situaciones en las que se mantenían en silencio aparecían como aspectos clave al momento de caracterizarse a sí mismos como alumnos.

Lo anterior se veía aun más profundizado por las dificultades que acarreaba la frecuente inasistencia de los docentes. Estas situaciones generaban fuertes críticas por parte de los alumnos, quienes se referían a ellas a lo largo de nuestras conversaciones, incidiendo en el posicionamiento que asumiríamos durante los encuentros pautados con ellos. Era claro que el equipo de investigación debía asumir como objetivo primordial la generación de confianza en cuanto al cumplimiento de lo pactado, no solo como una posición ética frente a los alumnos, sino también como una condición fundamental en la tarea de sostener el trabajo de campo y el logro de una posición alternativa para la construcción de "las voces" en el proceso de investigación.

Esto se vio reflejado, entre otras cosas, en el respeto por la palabra dada (tanto en las cuestiones organizativas grupales, como en los sentidos y posicionamientos con respecto al espacio y a la participación en el mismo). Los días, los horarios y las pautas que íbamos acordando con los alumnos eran rigurosamente respetados por el equipo de investigación, aun en los casos en que había muy pocas posibilidades de que se dictaran clases.

\section{Voces de lo Escolar y lo Extra-escolar}

Indagar los aprendizajes escolares y extraescolares de los alumnos a partir de la interpelación de sus voces permitió en un doble movimiento, un acercamiento a un variopinto grupo de concepciones e impresiones "nativas" sobre el tema, a la vez que promovió una forma alternativa de construcción del conocimiento. En el intento por desentrañar aquello que los jóvenes señalaban como 
rasgos diferenciales de los aprendizajes producidos en los diversos contextos por los cuales transitaban, fueron apareciendo una serie de caracterizaciones específicas de lo escolar y una serie de relaciones entre los diversos contextos.

En palabras de los alumnos, la principal diferencia entre aprender en la escuela y aprender fuera de ella se relaciona con la forma en la que están secuenciados los contenidos a aprender, es decir, por la forma en que la variable tiempo ordena el aprendizaje escolar. Existiría así una especie de contraposición entre el modo de aprender ordenado y secuenciado, propio del aprendizaje escolar, y el modo desordenado y caótico en que se aprendería fuera de la escuela. En palabras de una joven "[...] afuera lo aprendés mientras te está pasando, como que te va golpeando y acá es más estudiar y que te quede en la cabeza, estudiar para que me quede y después se me olvidó, y afuera no queda [...]". La variable tiempo no solo aparece como el elemento que diferencia el aprendizaje entre contextos, sino también como una variable que define el sentido mismo de estar en la escuela. El periodo escolar se reitera como un periodo de espera, de latencia y de aprendizaje para ser utilizado "después", en un futuro, pero con la paradoja que presenta la posibilidad del olvido de los contenidos allí aprendidos.

La explicación suele ser la forma en que el alumnado describe la forma de trabajo de sus docentes, aunque la misma no aparece como exclusiva del espacio escolar. Observar, probar, preguntar son algunas de las acciones que se señalan a la hora de caracterizar los aprendizajes extraescolares. No obstante, a la hora de describir las formas de aprender en sus espacios de tránsito cotidiano fuera de la escuela, en muchos casos se señala la existencia de sujetos con mayor experiencia que les van enseñando o los van guiando en las diferentes actividades que realizan.

Los jóvenes señalan que, a diferencia de lo que ocurre en la escuela (donde hay un cúmulo de saberes o contenidos programados a enseñar y un sentido asignado desde lo social o institucional), una gran proporción de aprendizajes que se producen en sus espacios extraescolares surgen espontáneamente, a partir de la interacción con los diversos grupos sociales o con los medios de comunicación.

Dentro del mismo espacio escolar aparecen formas de aprendizaje diferenciadas por los alumnos según diversos elementos. Entre otras cosas, distinguen entre aquellas clases en que "se aprende más" - aludiendo al hecho de sentirse interpelados y movilizados por otra persona- de aquellas en las que no se sienten interpelados o invitados para la realización de actividades de aprendizaje. Así, por ejemplo, una de las alumnas señalaba que “[...] entonces 
en esa clase sí, o sea, son trabajos en donde estamos en grupos de cuatro o cinco, pero donde tenemos que ir y hablar con el otro. Las otras materias son distintas, te dan un trabajo y listo [...]".

La distinción mencionada se ve relacionada, en primer lugar, con la forma en que se organiza la clase o con las características del profesor. Así, señalan "está buena porque explica bien y te hace trabajar en conjunto"; "[...] para mí él no explica bien... como que él ya lo sabe y piensa que lo vamos a entender como él lo entendió [...]”. En segundo lugar, se advierte que el interés por el trabajo con algún área en particular se relaciona también con el tipo de contenido que se proponía desde el espacio de la materia. Los alumnos describían como buenas clases a aquellas en las que los docentes se interesaban por promover que ellos hagan o hablen, es decir, a partir de la potencialidad de la acción.

A partir del contacto con estos grupos de jóvenes, se pudo ir visualizando que muchos de ellos, en sus vidas cotidianas, desarrollan un cúmulo de actividades propias de la vida adulta: trabajar, cuidar hermanos, ocuparse de las tareas del hogar, participar en diversas instituciones políticas, sociales o religiosas. Nos interesa destacar que las formas en que son calificados estos alumnos varían de acuerdo con el contexto. Así, se han observado casos de los denominados "malos alumnos", que en sus tareas u ocupaciones cotidianas se desenvuelven con absoluta responsabilidad y eficacia. En este sentido, cabe reflexionar críticamente en torno a las orientaciones que asumen las evaluaciones elaboradas desde la institución escolar, las cuales parecen "recortar" la integridad de las personas, adjetivándolas desde la óptica del rendimiento escolar.

Por lo expuesto, se podría comenzar a discutir con aquellas investigaciones y perspectivas teóricas desde donde se suele describir a los contextos escolares y extraescolares a partir de caracterizaciones sumamente diferenciadas. Este trabajo de investigación permite, en principio, señalar un conjunto de diferenciaciones dentro del mismo contexto escolar, a la vez que señalar una suma de continuidades entre los diversos contextos transitados por los alumnos, mostrando las distintas tonalidades de la experiencia humana, tanto en contextos escolares como en los extraescolares.

\section{Voces que Concluyen... y Abren Interrogantes}

El análisis de las características que adoptan los aprendizajes escolares y 
comienza a mostrar una serie resultados preliminares que suscitan nuevas reflexiones y preguntas.

Como se ha expuesto, la literatura preocupada por analizar las especificidades de los aprendizajes que se producen en los diversos contextos hace énfasis en las particularidades que adquieren los mismos, según el escenario donde se produzcan. Las indagaciones de nuestro trabajo de campo permiten, en principio, cuestionar las distinciones tajantes entre los aprendizajes en los diferentes contextos, como así también señalar que cada contexto puede contar con la existencia de diversos matices, rompiendo de algún modo con la idea de homogeneidad. Las formas de trabajo en que organizan sus clases los docentes, las características profesionales y personales de los mismos, entre otros, pueden ser, desde el punto de vista de los alumnos, elementos que marcan una heterogeneidad de características.

Estas observaciones no son menores, en tanto dan claras muestras de que al interior del contexto escolar los alumnos pueden participar de actividades/ tareas en las que pueden sentirse interpelados o no, mostrando posiciones más o menos activas o pasivas. Esta idea puede discutir con aquellas perspectivas teóricas para las cuales las posiciones de los alumnos en los espacios escolares son leídas en términos de pasividad únicamente.

Desde otro ángulo, elementos como el tiempo se tornan destacables en la reflexiones de los jóvenes en torno de ambos tipos de aprendizaje, aunque con rasgos claramente diferenciados. El tiempo del aprendizaje escolar aparece como aquello que determina la secuencia del aprendizaje, en tanto eje ordenador $-\mathrm{y}$ limitante- de aquello que se aprende. En los escenarios extraescolares, en cambio aparece como un elemento desordenado y no necesariamente condicionante de la actividad.

Asimismo, la explicación, como elemento que caracterizaría en principio el aprendizaje escolar desde el punto de vista juvenil, aparece también en las caracterizaciones que los jóvenes realizan de sus aprendizajes extraescolares. En estos últimos, la explicación se presenta a través de un sujeto con mayor nivel de experticia en la tarea o la guía de algún adulto.

Nuestras primeras impresiones para el análisis de los diversos tipos de aprendizaje se vieron acompañadas por una forma de abordaje metodológico donde las voces de los estudiantes fueron las privilegiadas. Consideramos necesario continuar reflexionando en torno a cuáles son las condiciones que posibilitan que los diarios de aprendizaje puedan desarrollarse y sostenerse en el tiempo, a fin de mostrar su potencial, no solo en cuanto a la investigación académica, sino también - y de manera muy singular - en términos de 
experiencias significativas desde el punto de vista de los alumnos participantes. En la experiencia desarrollada, el diálogo entre el contexto y las voces que allí aparecían permitió comprender que aspectos tales como la confianza o la flexibilidad en la propuesta de escritura eran condiciones necesarias para sostener el trabajo. El "campo" impone sus propias condiciones frente a nuestra inquietud por conocerlo, pero también implica la posibilidad de interpelación de la propia práctica de investigación e intervención docente.

En cuanto a la participación de los alumnos, puede decirse que el hecho de haber promovido espacios de escritura y discusión abrió la posibilidad de hablar sobre ellos - y entre ellos - en un espacio en que la comunicación entre adultos y adolescentes parecía estar signada por el silencio. En tanto partícipes de la pregunta de investigación, el trabajo realizado abrió la posibilidad de que también el alumnado reflexionara respecto de los procesos compartidos, como aprendices de una cultura que trasciende los muros escolares. Seguramente, tanto este campo de estudios como la posibilidad de dialogar, intentando superar las tradicionales "perspectivas adultocéntricas" (Chaves, 2005) puede ayudarnos a despejar un cúmulo de problemas concretos en el ámbito de las experiencias escolares y producir un conocimiento social a partir de un posicionamiento crítico de nuestros entornos, así como de nosotros mismos. Queda claro que aspectos tales como la autoestima, la motivación para aprender e investigar, la capacidad de discusión y participación en grupo, entre otros, conforman la compleja trama de los procesos que forjan las subjetividades y, por tanto, tienen un fuerte impacto en la vida presente y futura de los sujetos. Solo a través del cuestionamiento de ciertas lógicas tradicionales, ligadas, entre otras, a la idea de incompletitud del alumno, a la importancia otorgada a su futuro más que a su presente, a la explicación del docente más que a sus formas de concebir el mundo (Skliar, 2005) podremos ofrecer nuevos escenarios escolares y extraescolares a nuestros alumnos y, seguramente, promover y aprovechar el potencial transformador que germina en sus voces, pero también en sus silencios.

La participación de los alumnos en esta investigación devino en un modo posible de pensar la problemática abordada. En primer lugar, puso en discusión las propias concepciones de sus docentes, traducidas en sus prácticas pedagógicas de enseñanza, mientras que, en segundo lugar, problematizó las producciones teóricas y las políticas educativas que señalan la apatía o la falta de interés de los adolescentes, mostrando sensaciones y valoraciones que, en tanto tales, no suelen ser relevadas o apropiadas por ninguna instancia de toma de decisiones. Por otro lado, puede afirmarse que la investigación con- 
tribuyó a comprender la forma en que se construye el conocimiento en torno al tema abordado, generando nuevos interrogantes $-\mathrm{y}$ reformulando otros ya clásicos-, entre los que podemos mencionar los siguientes: qué aspectos obstaculizan y qué aspectos pueden facilitar los posicionamientos críticos y reflexivos por parte de los docentes y los alumnos; cuáles son las intervenciones del equipo de investigación que contribuyen -y cuáles obstaculizan - al "potencial transformador" de las voces del estudiantado; qué instrumentos de indagación, por un lado, y qué tipo de actividades escolares, por el otro, resultan convenientes a los fines de conocer las voces de los alumnos; en qué medida dichos instrumentos pueden ser generalizables a otras instituciones, y en qué medida requieren de la formulación y reformulación permanente, de acuerdo con cada institución y grupo escolar; qué lugar ocupan y qué lugar deberían ocupar los conocimientos extraescolares en contextos escolares, y viceversa; cuáles son las corrientes, categorías y conceptualizaciones más pertinentes para potenciar nuevas líneas de trabajo.

Algunos campos disciplinares concentran su atención en la individualización de las conductas, mientras que otros lo hacen desde perspectivas mucho más macropolíticas, de acuerdo con concepciones más o menos funcionalistas, más o menos relativistas, etc. Lo que muestran nuestras tareas de campo, como así también los estudios que abordan temas afines al nuestro, es la necesidad de compartir distintas perspectivas, indagando (problematizando) nuestras prácticas de investigación a la vez que las estamos ejerciendo; produciendo conocimiento en acción, a la vez que intentamos romper con algunas de las dicotomías tradicionales, del tipo sujeto/objeto, medios/fines, texto/contexto, interpelando, en definitiva, nuestros propios supuestos teórico-metodológicos en pos de una reflexión colectiva. En este sentido, los aportes que han realizado las perspectivas psicológicas, las etnográficas, las sociológicas y las provenientes de los estudios del lenguaje y el discurso en torno de aproximaciones metodológicas cada vez más ajustadas a las indagaciones empíricas de nuestro interés, resultan evidentes. Consideramos que, en la actualidad, ninguno de los campos agota por sí solo los problemas conceptuales y empíricos que las nuevas situaciones educativas involucran. A diferencia del paradigma instalado desde los orígenes de las ciencias y las instituciones modernas, las formas de acceso al conocimiento son hoy múltiples y susceptibles de ser abordadas desde puntos de vista muy diferentes.

Ya no se trata de valorar y trasmitir las certezas y componentes "objetivos" de nuestra realidad, sino de cuestionarlos, para construir nuevos temas y abordajes, en contextos de incertidumbre. Y es aquí donde, nuevamente, 
surge la (in)quietud de los silencios. Quizás, de la mano de los procesos mencionados, sea este el momento de empezar a romper con las figuras de pasividad que, desde el paradigma de las ciencias sociales de los siglos XIX y XX, se han asociado a las situaciones en que la palabra dicha se encuentra ausente. Mientras que los científicos sociales se han ocupado más frecuentemente por la "evidencia" de la palabra y la racionalización de la comunicación, se destaca aquí la necesidad de continuar y fortalecer las líneas de trabajo que revaloricen la situación de silencio como instancia de indagación en sí misma. Ante las dificultades de los adolescentes de expresar sus sentimientos, emociones y apreciaciones más profundas en torno de los escenarios y actores con los que vivencian sus experiencias, podemos, como adultos interesados en la cuestión social y educativa, contribuir a la comunicación intergeneracional, pero también intrageneracional, comenzando por resignificar y escuchar la palabra "no evidente". La incomodidad que antes se generaba da paso, entonces, a miradas respetuosas de los tiempos y situaciones personales de cada sujeto, a favor de un proceso colectivo de gran potencial, tanto para investigadores como para cada uno de los grupos en los que se interviene.

Seguramente esto contribuirá a la generación de sentidos, singulares y contingentes, diferentes de los significados "dados" por las ciencias modernas, pero mucho más potentes en lo que refiere a la necesaria reflexión que nos debemos los adultos como educadores. En este sentido, los aportes de algunas reflexiones filosóficas interesadas por lo educativo también se tornan necesarias. En palabras de Bárcena (2005), una sensibilidad tal

[...] nos habla del aprendizaje de la incertidumbre y de la importancia de perder el miedo a vivir y pensar sin certezas absolutas. En definitiva, nos habla de un aprendizaje de la experiencia [...] La incertidumbre pedagógica puede vivirse como un obstáculo para el pensamiento y como una parálisis en la acción. Y, sin embargo, [...] constituye el principal dispositivo para implicarnos en una experiencia reflexiva dentro de las situaciones prácticas de la educación. (pp. 25-29)

Según este mismo autor, la significación alude a un sentido previamente formulado e identificado, mientras que el sentido implica la apertura a posibles y nuevas significaciones.

Es en este marco, un tanto incierto y, por eso mismo, "potente" y sumamente interesante, que nos planteamos nuestra perspectiva de futuros proyectos como un gran desafío para pensar nuevos desarrollos en el campo 
educativo. Esto involucra nuestro interés por fomentar posicionamientos crecientemente activos de parte de los sujetos involucrados en nuestras intervenciones, con miras a dirigir nuestro pensamiento y nuestra acción en busca de nuevas "aperturas" más que en el logro de ideas prolijamente cerradas. 


\section{Referencias}

Andrews, J. y Yee, W. (2006). Children's "funds of knowledge" and their real life activities: Two minority ethnic children learning in out-of-school contexts in the UK. Educational Review-Special Issue, 58(4), 435-449.

Aries, P. (1986). La infancia. Revista de Educación, 281, 5-17.

Bárcena, F. (2005). La experiencia reflexiva en educación. Barcelona: Paidós.

Bernstein, B. (1971). Class, codes and control (vol. 1). Londres: Routledge and Kegan Paul.

Borzone, A. M. y Rosemberg, C. (2000). Leer y escribir entre dos culturas. El caso de las comunidades kollas del Noroeste Argentino. Buenos Aires: Aique.

Candela, A. (2006). Del conocimiento extraescolar al conocimiento científico escolar: un estudio etnográfico en aulas de la escuela primaria. Revista Mexicana de Investigación Educativa, 30, 797-820.

Carraher, T.; Carraher, D. y Schlieman, A. (1998). En la vida diez, en la escuela cero. México: Siglo XXI.

Cazden, C. (1991). El discurso en el aula. El lenguaje de la enseñanza y del aprendizaje. Barcelona: Paidós.

Certeau, M. de (1979). La invención de lo cotidiano. México: Universidad Iberoamericana.

Chaves, M. (2005). Los espacios urbanos de jóvenes en la Ciudad de La Plata (tesis de doctorado). Universidad Nacional de La Plata, Buenos Aires.

Cimolai, S.; Lucas, J. y Pérez, A. (2011). Relaciones entre aprendizajes escolares y extra-escolares en la investigación educativa. Fundamentaciones, tendencias y perspectivas. Electronic Journal of Research in Educational Psychology, 9, (1), 383-414.

Elichiry N. E.; Maddonni P.; Scavino, C.; Aizencang, N.; Arrúe, C.; Bendersky, B. et al. (2008). Sujetos, familias y escuelas: Continuidades y discontinuidades en los aprendizajes cotidianos. Ponencia presentada en las XV Jornadas de Investigación.

Elichiry, N. E.; Scavino, C. y Arrúe, C. (2005). Deberes escolares ¿un nexo entre la familia y la escuela? Revista Argentina de Educación, 29, 87-100. 
Ferreiro, E. (1986). El cálculo escolar y el cálculo con dinero en situación inflacionaria. En E. Ferreiro, Proceso de alfabetización. La alfabetización en proceso. Buenos Aires: Centro Editor de América Latina.

Ferreiro, E. y Teberosky, A. (1979). Los sistemas de escritura en el desarrollo del niño. México DF: Siglo XXI.

Fielding, M. (2004). Transformative approaches to student voice: Theoretical underpinnings, recalcitrant realities. British Educational Research Journal, 30(2), 295-311.

Fielding, M. y Bragg, S. (2003). Students as researchers: Making a difference. Cambridge: Pearson Publishing.

Gandulfo, C. (2007). Entiendo pero no hablo. El guaraní "acorrentinado" en una escuela rural: usos y significaciones. Buenos Aires: Antropofagia.

Gélis, J. (1994). La individualización del niño. En P. Aries y G. Duby (Dir.), Historia de la vida privada (pp. 311-329). Barcelona: Taurus.

Harris, A. y McGregor, J. (2007). Prefacio. En Rudduck, J. y Flutter, Cómo mejorar tu centro escolar dando la voz al alumnado. Madrid: Morata.

Hughes, M. (2006a). End of award report: Home-school knowledge exchange and transformation in Primary Education. Londres: ESRC-TLRP. Recuperado de http://www.esrcsocietytoday.ac.uk

Hughes, M. (2006b). Home-school knowledge exchange. Educational Review, 58(4), 385-487.

Jasis, P. y Ordoñez-Jasis, R. (2004). Convivencia to empowerment: Latino parent organizing at La Familia. The High School Journal, 88(2), 32-42.

Kaplún, G. (2004). Culturas juveniles y educación: pedagogía crítica, estudios culturales e investigación participativa (la cumbia villera y concheta, el rock de la calle y la escuela). En Los jóvenes: múltiples miradas (pp. 3-13). Neuquén: UNC.

Labov, W. (1969). The logic of non-standard English. En J. E. Alacis (Ed.), Report of the twentieth round table meeting on linguistics and languaje studies (pp. 1-29). Washington: Georgetown University Press.

Limón M. y Carretero M. (1997). Las ideas previas de los alumnos ¿Qué aporta este enfoque a la enseñanza de las ciencias? En M. Carretero (Ed.), Construir y enseñar. Las ciencias experimentales (pp. 19-46). Buenos Aires: Aique. 
Macbeath, J.; Demetriou, H.; Rudduck, J. y Myers, K. (2003). Consulting pupils: A toolkit for teachers. Cambridge: Pearson Publishing.

McGregor, J. (2005). Students as researchers. Cranfield: National College for School Leadership.

Milstein, D. (2006). Y los niños, ¿por qué no?: Algunas reflexiones sobre un trabajo de campo con niños. Avá, 9, 49-59. Recuperado de http://www. scielo.org.ar/scielo.php?

Moll, L. y Greenberg, J. (1990). Creating zones of possibility. En L. C. Moll (Ed.), Vygotsky and education: Instructional implications and applications of sociohistorical psychology (pp. 319-348). New York, NY: Cambridge University Press.

Rudduck, J. (2006). The potential of listening to pupils. Educational Review, 58(2), 131-231.

Rudduck, J. y Flutter, J. (2007). Cómo mejorar tu centro escolar dando la voz al alumnado. Madrid: Morata.

Rudduck, J. y McIntyre, D. (2007). Improving Learning through Consulting Pupils. Abingdon, UK: Routledge.

Skliar, C. (2005). Poner en tela de juicio la normalidad, no la anormalidad. En P. Vain y A. Rosato (Coords.), Comunidad, discapacidad y exclusión social. La construcción social de la normalidad. Alteridades, diferencias y diversidad. Buenos Aires: Novedades Educativas.

Stenhouse, L. (1991). Investigación y desarrollo del currículum. Madrid: Morata. Wells, G. (1986). La experiencia del lenguaje de niños de cinco años en la casa y en la escuela. En J. Cook (Ed.), La construcción social de la alfabetización (pp. 85-108). Buenos Aires: Paidós.

Manuscrito recibido: 10 de enero, 2011

Revisado: 24 de marzo, 2011

Aceptado: 16 de mayo, 2011 